\title{
STANCE PHASE CONTROL OF ABOVE-KNEE PROSTHESES: KNEE CONTROL VERSUS SACH FOOT DESIGN
}

\author{
JEFFREY L. STEIN \\ Department of Mechanical Engineering and Applied Mechanics, The University of Michigan, \\ Ann Arbor, MI, U.S.A. \\ and \\ WOODIE C. FLOWERS \\ Department of Mechanical Engineering, Massachusetts Institute of Technology, Cambridge, MA, \\ U.S.A.
}

\begin{abstract}
The mobility of above-knee amputees $(\mathrm{A} / \mathrm{K})$ is limited, in part, due to the performance of $\mathrm{A} / \mathrm{K}$ prostheses during the stance phase. Currently stance phase control of most conventional $\mathrm{A} / \mathrm{K}$ prostheses can only be achieved through leg alignment and choice of the SACH (Solid Ankle Cushioned Heel) foot. This paper examines the role of the knee controller in relation to a SACH foot during the stance phase of level walking.

The three-dimensional gait mechanics were measured under two stance phase conditions. In the first set of trials, the amputee used a prosthesis with a conventional knee controller that allowed the amputee to maintain the knee joint in full extension during the stance phase. In the second set of trials, the prosthetic knee, during stance, echoed the modified kinematics of the amputee's sound (intact) knee that had been recorded during the previous sound stance phase.

Analysis and interpretation of the data indicate the following: (1) SACH foot design can strongly influence the walking mechanics independent of the knee controller; (2) knee controller design and SACH foot design are mutually interdependent; and (3) normal kinematics imposed on the prosthetic knee does not necessarily produce normal hip kinematics (e.g. reduce the abnormal rise in the prosthetic side hip trajectory). Future research is necessary to explore and exploit the interdependency of prosthetic knee control and foot design.
\end{abstract}

\section{INTRODUCTION}

The highest amputee/prosthesis interaction forces during above-knee $(\mathrm{A} / \mathrm{K})$ amputee level walking occur during the prosthetic stance phase. As a result the stance phase is the most physically demanding phase on the amputee and the most technologically challenging to the prostheses designer.

There have been many attempts to improve the performance of $A / K$ prostheses during the stance phase of level walking (Judge, 1980). These attempts have either been focused on knee controller design or on foot/ankle design. The aim of this study is to determine if the design of the prosthetic knee joint controller and the design of the foot and ankle assembly are interdependent.

The interdependency of knee controller design and SACH foot design, can be studied by comparing the gait of an $A / K$ amputee resulting from using a prosthesis with two different knee controllers but the same foot and ankle assembly. The differences in the knee controllers should produce large enough differences in the gait mechanics to permit separating the contributions of the knee dynamics from the foot and ankle dynamics to the overall gait mechanics. The two knee controllers chosen for this study are a conventional (CL) controller and a prototype controller called

Received 22 May 1984; in revised form 6 May 1986. modified echo (ME) control. The foot and ankle unit used in this study is called a SACH foot.

\section{Foot and ankle design}

A typical conventional $\mathrm{A} / \mathrm{K}$ prosthesis has a single axis knee joint and a SACH foot. Other knee, ankle and foot mechanisms are available, but they are not as widely used. During the stance phase until knee break (KB) the prosthesis provides stable support for the amputee. The knee joint is forced against its mechanical hyperextension stops by the appropriate combination of hip moment, body placement and sound leg push-off (Stein, 1983). The SACH foot provides motion in the anteroposterior plane simulating plantar and dorsiflexion of the ankle and compensating, to some degree, for the lack of knee flexion. In addition the SACH foot simulates the extension of the toe.

The SACH foot has a compliant heel and toe section. These sections are manufactured with characteristics known as 'soft', 'regular' and 'stiff'. The stance phase gait mechanics can be altered by the choice of heel and toe stiffness and the alignment of the SACH foot on the shank.

\section{Conventional control}

The CL knee controller is an angle dependent nonlinear damper. During single support stance phase [prosthetic heel contact (PHC) to sound heel contact (SHC)] the amputee holds the conventional controlled prosthesis against the hyperextension stops as previously described. After SHC, which corresponds, 
approximately, to knee break, the amputee prepares for the swing phase by imparting cnergy into the prosthesis through his stump. The knee control unit dissipates enough of this energy so that the initiation of swing phase is correct. During the swing phase the damping action allows the prosthesis to move in an approximately normal kinematic manner. The damping scheme used in this study during the swing phase is identical to the one used by Grimes (1979) and was developed and tuned to allow the laboratory prosthesis simulator to behave in a functionally equivalent way to the amputee's everyday conventional prosthesis. The damping profile used is an angle dependent velocity squared damper.

\section{Modified echo control}

This control scheme was developed by Grimes and Flowers (1982), Grimes (1979) and implemented here with only small technical changes by the first author (Stein, 1983).

ME control is implemented from PHC to SHC. During this time, the knee angle of the prosthesis follows a trajectory which is modified from the trajectory recorded from the sound knee during the previous sound stance phase. From SHC to PHC the prosthesis is controlled as a damped pendulum exactly as described for the CL controller. The approach is called modified echo control because the sound knee profile must be adjusted so that it approximates a normal knee trajectory. There are two reasons why the sound knee trajectory must be modified: (1) The $A / K$ amputee flexes his sound knee more than the knee of a normal during the stance phase of level walking (Grimes, 1979) and (2) the maximum stance phase knee flexion is a function of cadence in normals (Grimes, 1979). Thus, the sound knee profile is multiplied by a gain factor which is chosen so that the maximum knee flexion angle of the modified sound trajectory matches the maximum knee flexion angle for non-amputee gait at the existing cadence. Cadence is determined from the time of one step, PHC to PHC.

\section{Stilting as a gait parameter}

The knee flexion provided by the ME controller during stance should alter the typical patterns observed in conventional $\mathrm{A} / \mathrm{K}$ amputee gait. This study will examine the vertical height in the anteroposterior plane of the hip during stance. The maximum height of the hip on the prosthetic side is greater during stance for $A / K$ amputees than for normals of comparable size. This effect is produced by the prosthesis characteristics and is called stilting.*

The gait parameter chosen in this study should be

\footnotetext{
* The term vaulting is sometimes used to describe this phenomenon. However, vaulting (along with the term hiking) is also used to describe the abnormal rise in the hip on the sound side resulting from the amputee trying to ensure toe clearance during prosthetic swing. The term stilting is introduced by the authors to avoid ambiguity.
}

sensitive to the prosthesis kinematics and dynamics but not sensitive to gait characteristics such as walking speed. For example, stilting might alternatively be defined as the difference between the maximum in the hip trajectory and the height of the hip at PHC. This definition of stilting, however, would be, amongst other things, sensitive to the speed of walking. Longer stride lengths typically accompany a higher cadence which means that the leg angle (as measured from vertical) at $\mathrm{PHC}$ would be larger than the leg angle at PHC for lower walking speeds. Therefore the hip height would be lower and the resulting difference between the maximum hip height and hip height at PHC would be greater. Therefore, this definition of stilting would make it an inappropriate choice as a gait parameter.

The hypotheses being tested by this research are: (1) stilting, defined as the maximum hip height on the prosthetic side during stance, is a gait parameter sensitive to the knee joint and SACH foot characteristics, (2) the ME knee controller will reduce stilting, (3) knee controller design and $\mathrm{SACH}$ foot design are interdependent.

In summary, two stance phase knee controllers will be tested. The first is a CL controller (swing and stance) and the second is an ME controller which is designed to produce kinematics of the prosthetic knee during the stance phase that are similar to normal gait. The swing phase is the same for both controllers. It is assumed that by providing the prosthesis with normal knee kinematics the ME controller will reduce stance phase stilting by 'aiding' the SACH foot's role of providing simulated knee flexion.

\section{MATERIALS AND METHODS}

The main objective of the walking trials was to collect gait data using a Selspot-based gait acquisition system known as TRACK (Antonsson, 1982) while an A/K amputee used the 'MIT Knee' simulator prosthesis in the Eric P. and Evelyn E. Newman Laboratory for Biomechanics and Human Rehabilitation at MIT. The laboratory is $18 \times 9$ $\times 3.5 \mathrm{~m}$ with a Kistler Forceplate located approximately at the midpoint of an $18 \mathrm{~m}$ walkway. Two sets of trials were run. During the first set of trials the simulator was controlled to simulate a conventional prosthesis and for the second set the simulator during prosthetic stance was controlled using modified echo control. This experimental set-up required two minicomputers running synchronously. One recorded the global three-dimensional kinematics and foot/floor forces, while the other controlled the prosthesis simulator and recorded data from its transducers (Stein, 1983; Stein and Flowers, 1984).

\section{Person-interactive prosthesis simulator}

The $\mathrm{A} / \mathrm{K}$ prosthesis simulator system used is shown in Fig. 1. This system was developed by Flowers and 


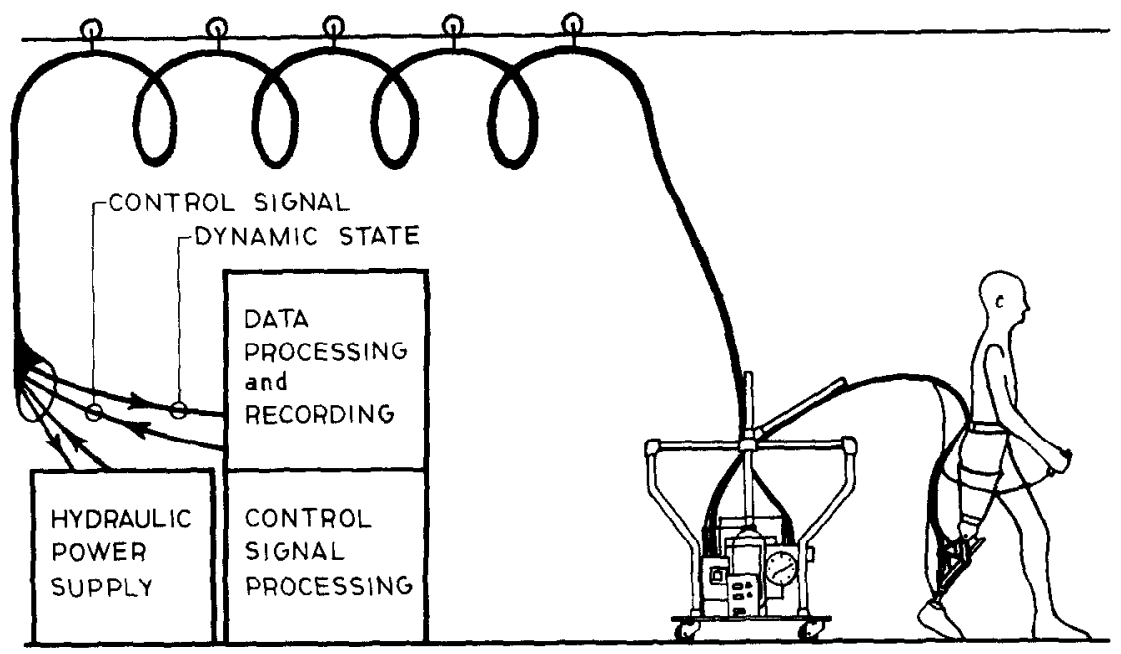

Fig. 1. Person interactive simulator system.

Mann (1977) and Flowers (1972) and was revised by Grimes (1979) and Stein (1983) (these references contain a complete description of the system). The dynamic and static characteristics of the knee joint of the prosthesis are controlled by a high performance servovalve. This servovalve controls the flow of 6.9 million $\mathrm{n} \mathrm{m}^{-2}$ (1000 psi) hydraulic fluid through an actuating ram. The servovalve is controlled by a digital computer receiving signals from the prosthesis and amputee. The feedback signals include: prosthetic knee moment, prosthetic and sound knee angles, $\mathrm{SACH}$ and sound foot/floor contact (heel and toe), and axial load in the prosthesis shank. A SACH foot is attached to the simulator and aligned in a conventional manner.

\section{Measuring amputee gait}

The three-dimensional kinematics and the foot floor forces were recorded during the walking trials. The kinematics were recorded by a Selspot I system which recorded the position and rotation of an array of infrared light emitting diodes (LEDs) attached to the shank (see Fig. 2). The kinematics of the thigh were measured by data provided by a knee angle potentiometer combined with the shank kinematics and knowledge of the geometric constraint imposed by the knee joint. This technique for measuring the thigh kinematics does not account for relative motion between the stump and socket. To date this motion has not been measured and is assumed to have a negligible effect on the determination of the hip trajectory (Bresler et al., 1957; Eberhart, 1947; Frigo and Rodano, 1982). The foot/floor forces were recorded with a Kistler force plate.

All of the position and force sensors were sampled at $157.5 \mathrm{~Hz}$ and then filtered with a well damped, doublepass, digital filter (Stein, 1983). A cutoff frequency of $5 \mathrm{~Hz}$ was used on all position data while a cutoff frequency of $20 \mathrm{~Hz}$ was used on the force information

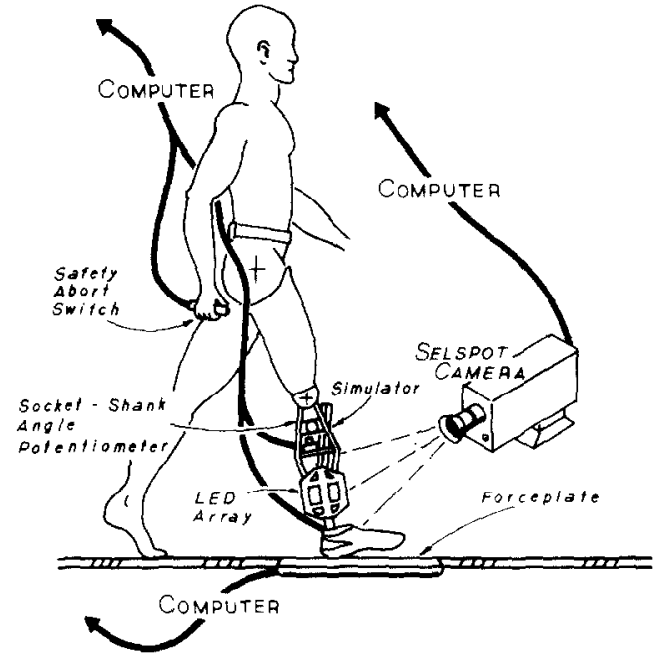

Fig. 2. Instrumented person interactive simulator.

except for the vertical foot/floor reaction force which was not filtered after being digitized.

All of the sensors used were calibrated. Position and rotational accuracy were $\pm 1 \mathrm{~mm}$ and $\pm 1.2^{\circ}$ respectively. The manufacturer's specification for the forceplate is $0.3 \%$ over the load range of the platform.

\section{Walking trial conditions}

One subject was tested over a six month period. A total of seventeen experimental sessions each lasting 1.5-3 h were conducted. A duplicate of the amputee's everyday SACH foot was used with the simulator (Otto Bock, Pedilan Soft Heel, Size 11) and the amputee's prosthesis alignment was also duplicated on the simulator. The subject was asked to walk at a comfortable pace and was given time to acclimatize before data was taken or the ME controller activated. 
The subject was generally unaware of where the forceplate was located and which trials were data collecting trials.

\section{Subject}

The subject who participated in this study is a left, unilateral $\mathrm{A} / \mathrm{K}$ amputee with no other health complications. He is a 26 yr old male, $1.7 \mathrm{~m}$ tall, weighs $68 \mathrm{~kg}$ (including the $5.7 \mathrm{~kg}$ prosthesis simulator) and has been an amputee since age 12. He wears a prosthesis with a Mauch S-N-S knee unit and an Otto Bock SACH foot with a soft heel. He is an active person and a good walker.

\section{RESULTS AND DISCUSSION}

\section{Data format}

In order to compare the results of different walking trials, the time scale was normalized as a percentage of the prosthetic stance phase. Zero percent corresponds to $\mathrm{PHC}$ while $100 \%$ corresponds to prosthetic toe off (PTO). Note PHC and PTO are measured by the forceplate. In addition, the timing of the stance subphases sound toe off (STO), prosthetic foot flat (PFF), prosthetic heel off (PHO), KB and SHC are provided in the appropriate figures. This information is included as a series of blocks along the abscissa. The center of a block is located at the average value of the subphase while the width of a block indicates plus or minus one standard deviation. Since all the data are for one amputee with a left side amputation, all plots are for the left or prosthetic leg. Angular kinematics are shown in the body coordinate systems, (i.e. saggital plane), while forces and translational kinematics are in the global coordinate system, (i.e. the average plane of progression). These planes, in general, do not differ greatly but may be rotated with respect to one another about a vertical axis by as much as $15^{\circ}$ (Stein, 1983). The sign convention is shown in Fig. 3. Knee flexion is positive, forward displacement is defined as zero at the location of PHC, vertical displacement is zero at the floor. All figures contain a sample of the several trials to show data scatter. Parameters such as maximum hip height are calculated by averaging the parameter for each curve over all the curves shown. All variables are in the SI system of units except for angular displacement which is in degrees.

\section{General gait mechanics}

The amputee was allowed to adopt a natural cadence during the walking trials. The general gait timing parameters for the gait produced during these trials are shown in Table 1. These parameters include: cadence (number of steps per minute where step is defined as

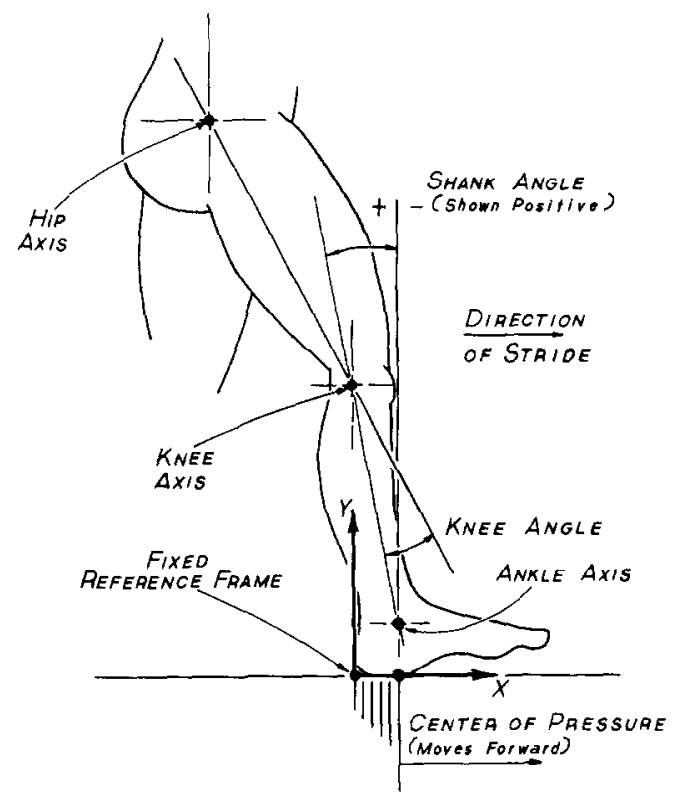

Fig. 3. Sign convention.

Table 1. Temporal gait factors as a function of knee characteristics-average value (S.D.).

\begin{tabular}{|c|c|c|c|c|c|}
\hline \multirow{4}{*}{$\begin{array}{l}\text { Temporal gait factor } \\
\text { Natural adopted cadence } \\
\text { Total prosthetic stance phase } \\
\text { time PHC to PTO }\end{array}$} & \multicolumn{4}{|c|}{ Controller } & \multirow{3}{*}{$\frac{\text { Units }}{{\text { Steps } \min ^{-1}}^{-1}}$} \\
\hline & \multicolumn{2}{|c|}{ CL } & \multicolumn{2}{|c|}{ ME } & \\
\hline & 100.0 & $(0.33)$ & 97.0 & $(0.82)$ & \\
\hline & 0.69 & $(0.023)$ & 0.71 & $(0.017)$ & $s$ \\
\hline Time from PHC to PFF & $\begin{array}{c}0.22 \\
32.0\end{array}$ & $\begin{array}{l}(0.012) \\
(1.7)\end{array}$ & $\begin{array}{l}0.15 \\
21.0\end{array}$ & $\begin{array}{l}(0.011) \\
(1.5)\end{array}$ & $\begin{array}{l}\mathrm{s} \\
\% \text { stance }\end{array}$ \\
\hline Time from $\mathrm{PHC}$ to $\mathrm{PHO}$ & $\begin{array}{c}0.34 \\
49.0\end{array}$ & $\begin{array}{l}(0.0097) \\
(1.4)\end{array}$ & $\begin{array}{c}0.24 \\
33.0\end{array}$ & $\begin{array}{l}(0.019) \\
(2.7)\end{array}$ & $\begin{array}{l}s \\
\% \text { stance }\end{array}$ \\
\hline Time from PHC to STO* & $\begin{array}{l}0.056 \\
8.1\end{array}$ & $\begin{array}{l}(0.010) \\
(1.5)\end{array}$ & $\begin{array}{c}0.10 \\
11.0\end{array}$ & $\begin{array}{l}(0.068) \\
(2.3)\end{array}$ & $\begin{array}{l}\mathrm{s} \\
\% \text { stance }\end{array}$ \\
\hline Time from $\mathrm{PHC}$ to $\mathrm{SHC}$ & $\begin{array}{l}0.60 \\
87.0\end{array}$ & $\begin{array}{l}(0.0020) \\
(0.29)\end{array}$ & $\begin{array}{c}0.62 \\
86.0\end{array}$ & $\begin{array}{l}(0.0053) \\
(0.74)\end{array}$ & $\begin{array}{l}\mathrm{s} \\
\% \text { stance }\end{array}$ \\
\hline Time from PHC to KB & $\begin{array}{c}0.50 \\
72.0\end{array}$ & $\begin{array}{l}(0.0035) \\
(0.50)\end{array}$ & $\begin{array}{c}0.57 \\
79.0\end{array}$ & $\begin{array}{l}(0.013) \\
(1.8)\end{array}$ & $\begin{array}{l}\text { s } \\
\% \text { stance }\end{array}$ \\
\hline
\end{tabular}

* The footswitch placement on the sound side shoe measures when the switch, not the shoe, is in contact with the floor. Toe off is particularly difficult to measure accurately without a second forceplate. 
PHC to SHC), time in prosthetic stance, average time from PHC to PFF, PHO, STO, SHC and KB. The results in Table 1 show that the CL controlled prosthesis produced gait with a higher cadence, a shorter stance phase and a longer single support phase as a percentage of the stance phase time. The ME controlled prosthesis produced a gait with a lower cadence, a more rapid transition to PFF, a longer foot flat period and a longer time in double support as a percentage of the stance phase time.

\section{Prosthetic knee angle}

The two knee controllers produced different prosthetic knee angle trajectories as shown in Fig. 4. Under $\mathrm{CL}$ control, the average knee joint is in full extension $5 \%$ after PHC and remains there until KB occurs at approximately SHC. Under ME control, the prosthetic knee angle at $\mathrm{PHC}$ has the same value $\left(3^{\circ}\right.$ of flexion) as the prosthetic knee angle under CL control. However, during the first $5 \%$ of stance, the knee angle extends to a maximum of $1^{\circ}$ of flexion and then starts to flex reaching a maximum of $14^{\circ}$ at $33 \%$. The knee then extends to within $5^{\circ}$ of full extension at SHC. At SHC the ME control algorithm becomes identical to the CL (passive) controller and the knee begins to flex in a manner similar to the trials using the $\mathrm{CL}$ controller exclusively, reaching approximately $30^{\circ}$ of knee flexion at PTO. The data scatter between trials is very small for the $\mathrm{CL}$ controller partly because the amputee has years of experience with it. The ME controller produces gait with more scatter (as indicated by knee angle trajectories). This is due to the amputee's lack of experience with this controller but is also a result of the prosthetic knee echoing the variations in the sound knee trajectories. The sound knee variations are con- sistent with variations reported by Eberhart (1947) and Winter et al. (1974).

\section{Center of pressure}

The graph of the center of pressure in the forward direction is a locus of points representing the instantaneous position on the floor where a single force vector alone (no moments) can represent the loading between the SACH foot and the ground. As the SACH foot rolls forward during stance, the center of pressure moves from the heel to the toe section of the SACH foot. Figure 5 shows a sample of trajectories for each controller. Four phases can be identified in both curves: (1) an initial period of small change in the center of pressure location with time (heel roll), (2) a phase of rapid change (foot flat), (3) a phase of very small change (initial toe roll), (4) a final phase following KB of moderate change (toe off).

One of the most outstanding differences between the trajectories is the point in time when the center of pressure begins to move rapidly forward (foot flat initiation). The ME controller causes the knee to flex and, therefore, rotates the shank forward. (The amputee maintains the thigh angle trajectory approximately independent of the knee controllers tested.) Thus foot flat is initiated sooner.

An important ramification of the center of pressure movement can be seen by plotting it as a function of shank angle. As seen in Fig. 6, there is little difference between the curves, thus indicating that the $\mathrm{SACH}$ foot could be modeled as a rolling cam. The cam's geometry then defines the center of pressure as a simple algebraic function of shank angle (see Fig. 7). This cam shape, as determined from Fig. 6, is related to the shape, alignment and physical properties of the heel and toe

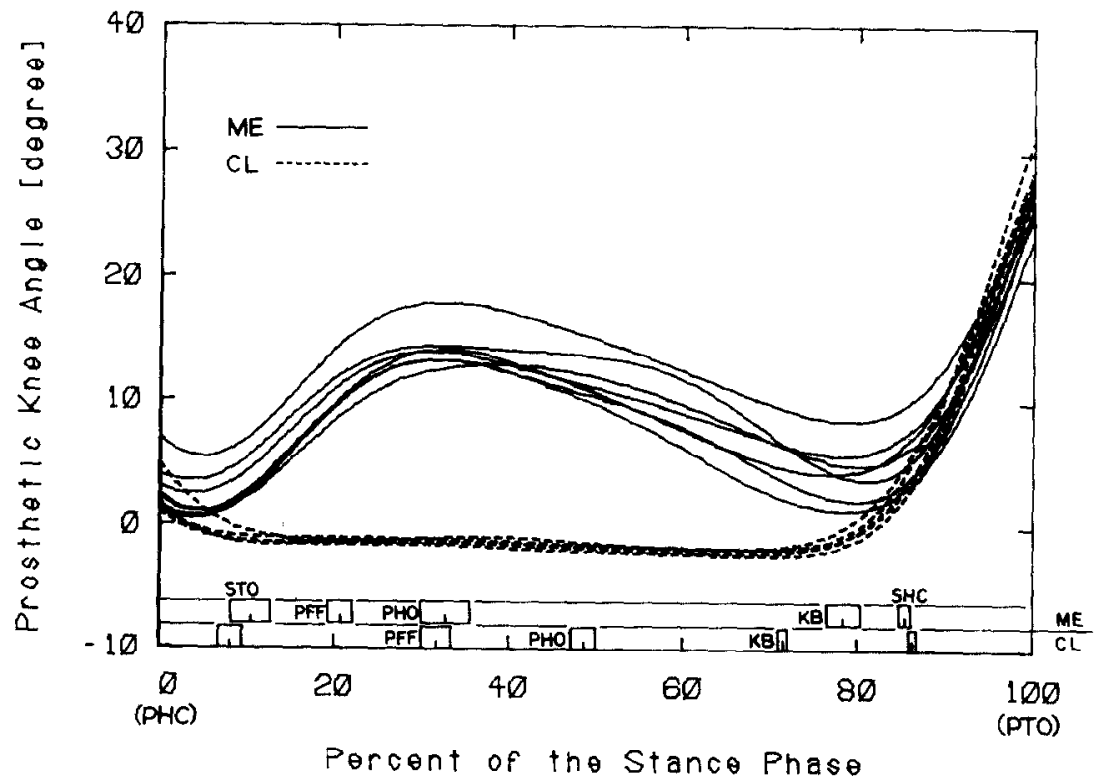

Fig. 4. A sample of prosthetic knee joint trajectories for the ME and CL stance phase knee controllers. 


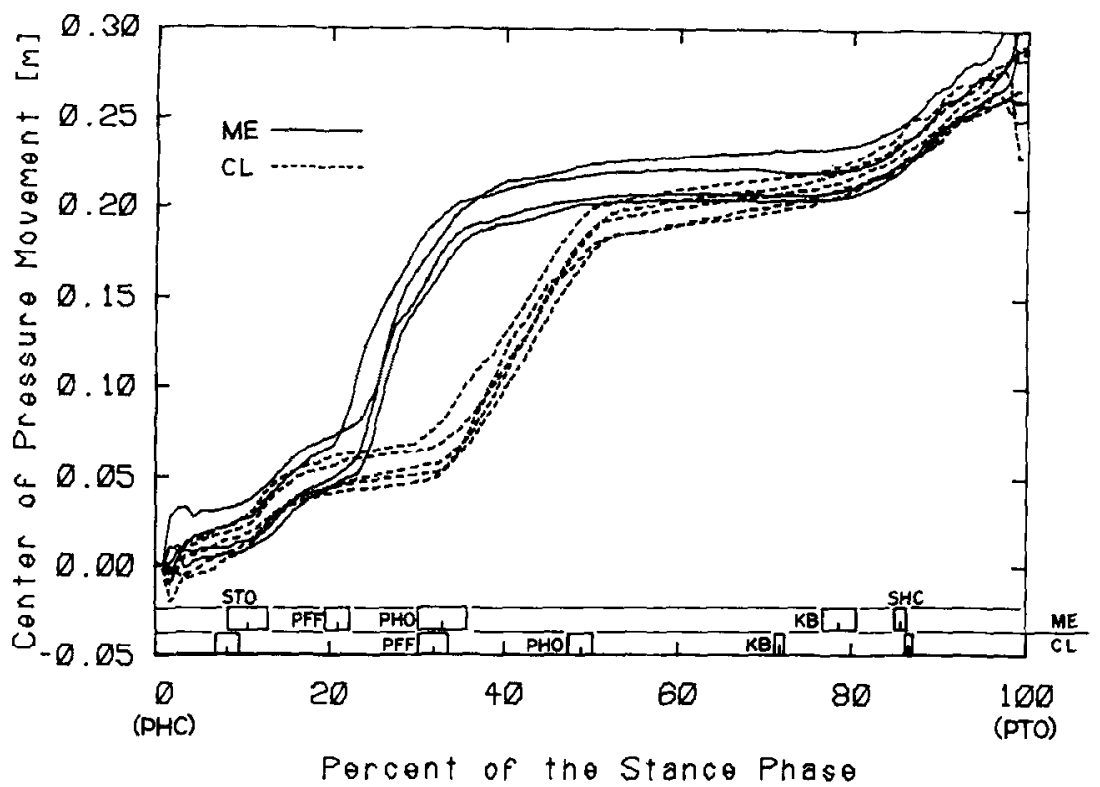

Fig. 5. A sample of center of pressure trajectories for the ME and CL stance phase knee controllers.

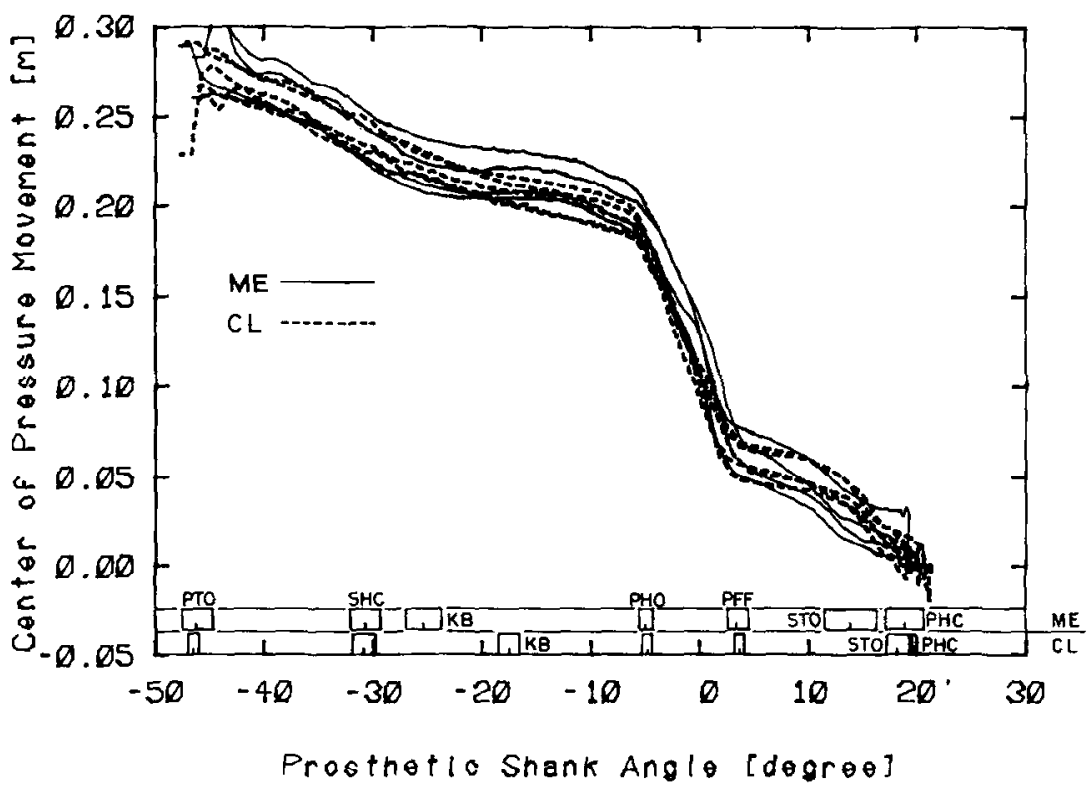

Fig. 6. A sample of center of pressure vs shank angle trajectories for the ME and CL stance phase knee controllers.

contact regions of the SACH foot and shoe. This implies that either the SACH foot loading is not altered by the knee controllers and thus the SACH foot deforms into the same shape during ambulation or the heel and toe stiffnesses are large enough relative to the loading on the foot that only insignificant changes in the shape of the SACH foot are produced during walking. Which of these possibilities is correct will be explored later in this paper. Independent of which explanation is correct, if the SACH foot can be modeled as a rigid cam for all prosthetic knee con- trollers then any alteration of knee angle during the stance phase would imply a constrained and, therefore, kinematically predictable hip trajectory.

\section{Hip displacement}

The hip displacement, as measured in this work, is really the displacement of a point rigidly attached to the suction socket at a location representing the hip joint. Thus, the assumption that the femur remains fixed with respect to the motion of the socket is used in presenting the following results. 


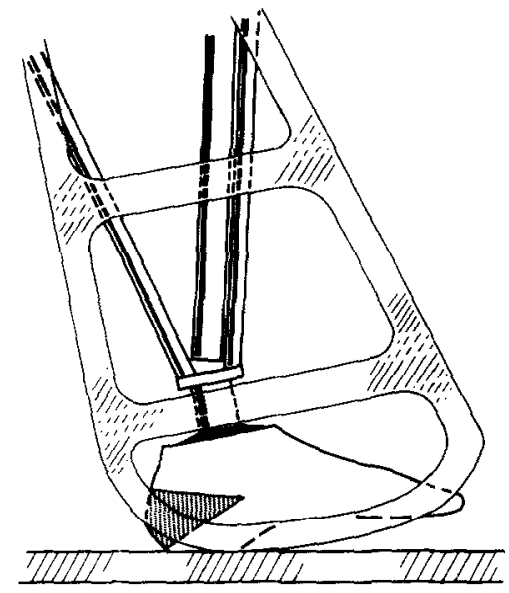

Fig. 7. The rigid cam profile model of the SACH foot.

The previous result implies that the hip kinematics produced during single support stance are determined by the SACH foot cam profile and the prosthetic knee joint trajectory. Because, in this study, the knee trajectories produced by the two controllers are known along with the SACH foot cam profile the role of the ME controller in affecting stilting can be determined. This section, therefore, will demonstrate whether controlling the knee joint to mimic a modified sound knee trajectory with this $\mathrm{SACH}$ foot is appropriate.

Stilting was defined previously as the maximum hip height on the prosthetic side attained during stance. The range of shank angles during which the maximum in the hip trajectories are observed is called the stilting range. This range is defined between the shank angles of $5^{\circ}$ before vertical and $25^{\circ}$ after vertical. This corresponds to 20 and $79 \%$ of stance for the ME controller and to 30 and $82 \%$ of stance for the $C L$ controller (see Fig. 8). Figure 8 shows that the maximum height of the hip trajectory is always greater in the trials using the ME controller. Specifically, the average hip heights for the trials shown are $0.909 \mathrm{~m}$ and $0.903 \mathrm{~m}$ with standard deviations of $0.00221 \mathrm{~m}$ and $0.00130 \mathrm{~m}$ for ME and CL controllers respectively. A statistical $t$ test yields a value of $t=5.45$, d.f. $=10$ and $p<0.001$. This means that there is greater than a $99 \%$ probability that the differences observed between the maximum hip heights are a result of real differences and not due to chance. The absolute height attained by the amputee is not reduced by flexing the knee during stance but rather it is increased. This can be explained by the analyzing the kinematics of the SACH foot and the prosthetic knee.

\section{Hip kinematics}

Hip height is determined by the vertical component of an imaginary stilt defined from the hip to the center of pressure of the foot/floor interaction force. The vertical component of this stilt is determined by its angular position and length. In general the length is a function of knee angle, SACH foot geometry and prosthesis compliance.

In this study there are some additional constraints that reduce the number of variables which affect the vertical hip height. First, as was previously shown, the SACH foot used can be represented by a cam. The cam profile defines a relationship between stilt length and stilt rotation. Second, the prosthesis compliance which is the compliance in the SACH foot (the stump/socket interactions were not measured) is not significant during the stilting period. This is shown in Figs 9 and 10. Figure 9 shows that the vertical height of the prosthetic knee joint during stilting is independent of the knee controller. This implies that either the vertical

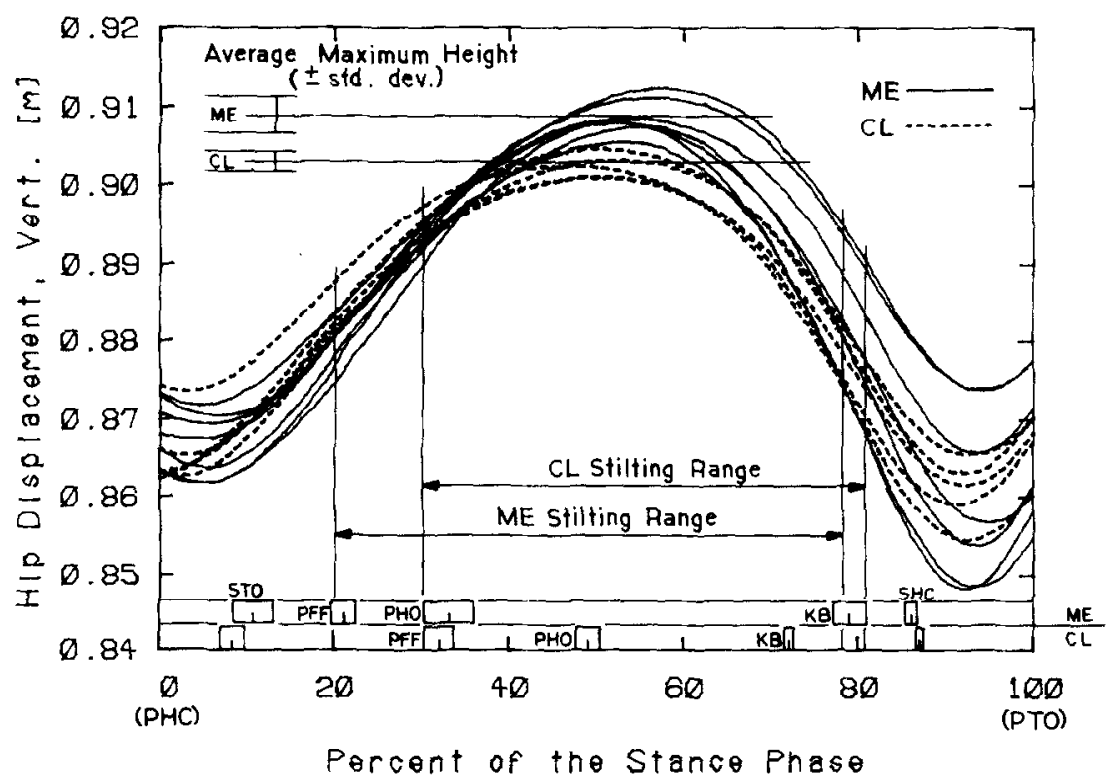

Fig. 8. A sample of vertical hip trajectories for the ME and CL stance phase knee controllers. 


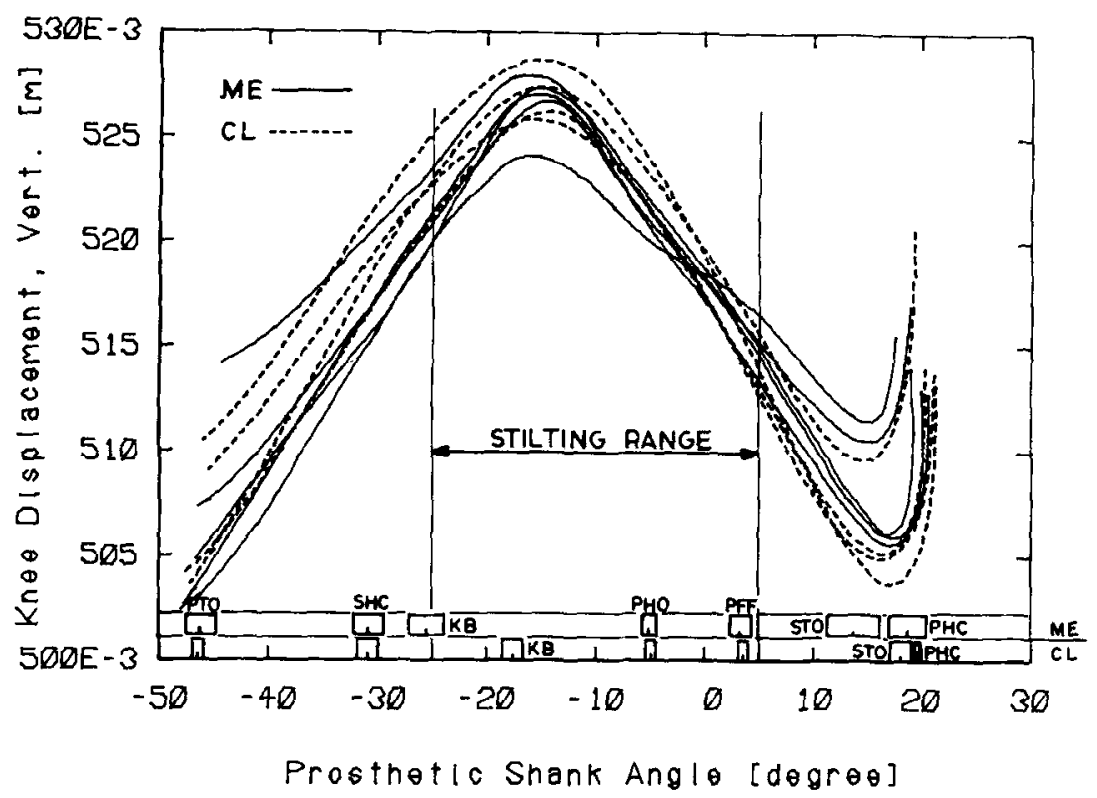

Fig. 9. A sampie of vertical displacements of the prosthetic knee joint as a function of shank angle for the $M E$ and $\mathrm{CL}$ stance phase knee controllers.

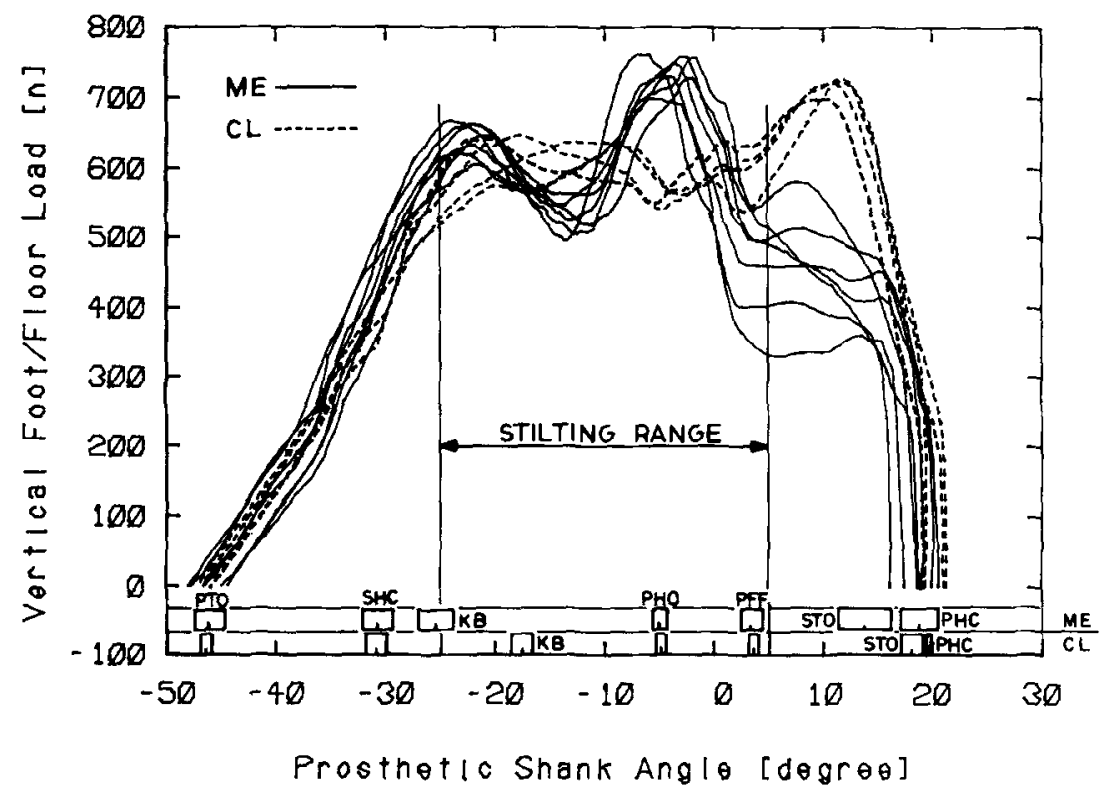

Fig. 10. A sample of vertical foot/floor forces as a function of shank angle for the ME and CL stance phase knee controllers.

load on the SACH foot as a function of shank angle is not changed by the knee controllers or that the local stiffness of the SACH foot is large enough to permit the assumption that the SACH foot is a rigid body. As shown in Fig. 10, the vertical loading is different for the two controllers in the stilting range. This fact supports the rigid (non-compliant) cam model of the SACH foot. Therefore, the independent factors which define the vertical hip height in this study are shank angle for the CL controller and shank angle plus knee angle for the $\mathrm{ME}$ controller.
The generation of the maximum in the vertical hip height trajectory is produced by the following kinematics. Figure 9 shows that there is only one maximum in the knee height trajectory and that it occurs at the non-zero shank angle of $16^{\circ}$ past vertical. This means, as can be seen in Fig. 6, that the center of pressure is under the toe section of the SACH foot (approximately $20 \mathrm{~cm}$ from where the heel contacted the floor). Therefore the largest vertical hip displacement will occur at this point in stance if the thigh is vertical. In order for the thigh to be vertical the knee angle must be 
at $16^{\circ}$ of flexion. Thus, as shown in Fig. 11, any physiologically reasonable amount of knee flexion (less than $32^{\circ}$ ) guarantees a higher hip displacement than would be produced with a knee angle of zero degrees (CL controller) for a shank angle of $16^{\circ}$ past vertical. The typical knee angle at this point in the gait cycle for the ME controller is approximately $12^{\circ}$.

However, the previous explanation does not guarantee that the highest point in the hip trajectory occurs at the highest point in the vertical knee trajectory. The actual peak in the vertical hip trajectory could occur at some other shank angle due to the particular combi-

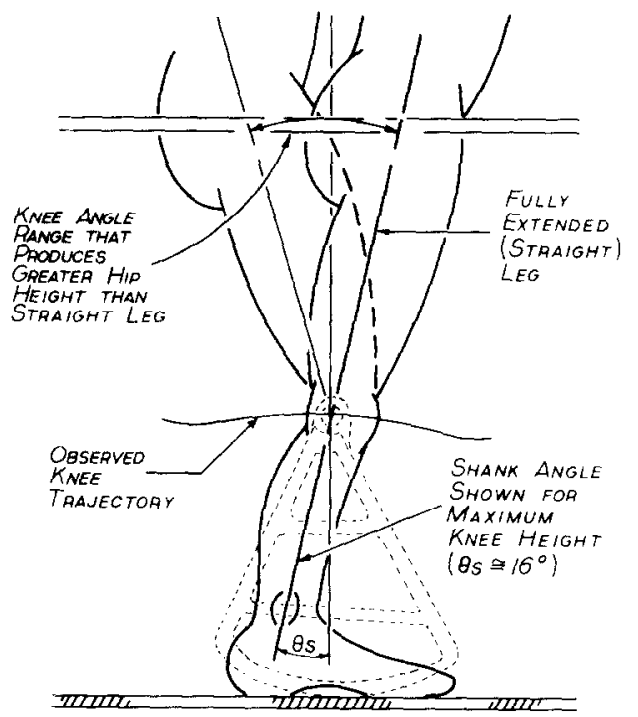

Fig. 11. The generation of hip kinematics from a variation in knee angles. nation of cam profile and knee angle trajectory. However, as shown in Fig. 12, the vertical hip trajectory reaches a maximum for the ME controller when the shank is approximately $12^{\circ}$ past vertical. This is close to the $16^{\circ}$ angle where the knee profile is maximum. This implies that with the 'sharpness' of the peak in Fig. 9 that the peak vertical hip trajectory is controlled by the $\mathrm{SACH}$ foot geometry. Figure 12 also shows that the $\mathrm{CL}$ controller produces the highest value in its trajectory at approximately $5^{\circ}$ past vertical. This is again consistent with the SACH geometry controlling the hip height trajectory. Thus any physiologically reasonable knee flexion angle implemented after $5^{\circ}$ past vertical will produce a vertical displacement trajectory with a peak value guaranteed to be greater than any produced with the zero angle $\mathrm{CL}$ controller.

The geometry, alignment and compliance of the $\mathrm{SACH}$ foot used in this study strongly influences the vertical displacement of the hip. If a novel knee controller, such as the ME controller, is going to produce 'improved' hip trajectories then the shankangle-center-of-pressure constraint must be violated. A SACH foot with a more compliant toe break section or other types of prosthetic foot and ankle units with compliant characteristics could alter this constraint.

\section{SUMMARY}

In summary, the prosthetic knee joint of an $A / K$ prosthesis was provided with kinematics similar to normals. The amputee's gait was compared to gait produced by the amputee when using a conventionally controlled knee joint. The maximum height of the hip

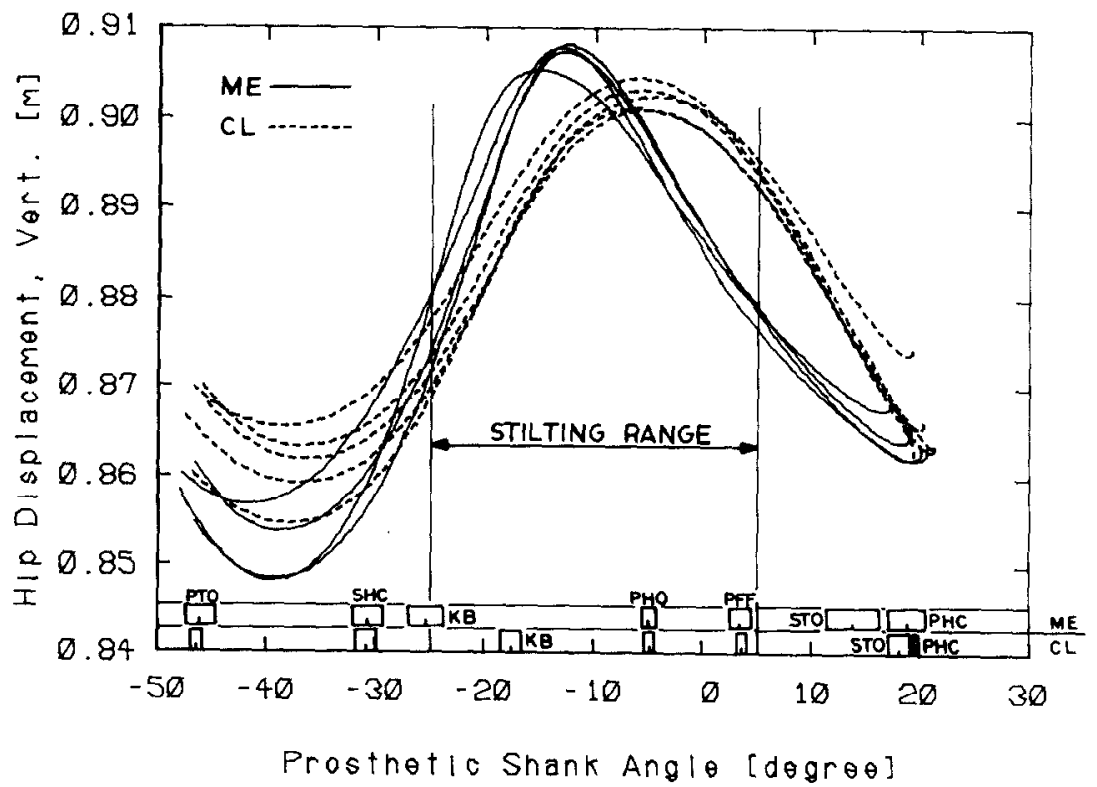

Fig. 12. A sample of vertical hip displacements vs shank angle for the ME and CL stance phase knee controllers. 
on the prosthetic side was increased when the knee joint was allowed to fiex. This was found to be a result of the particular combination of geometry, alignment and compliance of the SACH foot used. In particular, the trajectory of the center of pressure on the SACH foot was found to be a function of shank angle and invariant to the two different knee controllers tested. Therefore, to generate new hip kinematics requires a change in the SACH foot characteristics. While other factors are also important in evaluating the mobility of an $\mathrm{A} / \mathrm{K}$ amputee, clearly the $\mathrm{SACH}$ foot and knee controller must be designed interdependently to achieve a predictable change in the hip kinematics.

Acknowledgement-This research was supported by the National Science Foundation, grant ECS-8023193 and a Dupont University Science and Engineering Grant.

\section{REFERENCES}

Antonsson, E. K. (1982) A three-dimensional kinematic acquisition and intersegmental dynamic analysis system for human motion, $\mathrm{Ph}$.D. Thesis, Department of Mechanical Engineering, MIT, Cambridge, MA.

Bresler, B., Radcliffe, C. W. and Berry, Jr., F. R. (1957) Energy and power in the legs of above-knee amputees during normal level walking, Lower-extremity Amputee Research Project, Institute of Engineering Research, Univ. of California, Berkeley, Series 11, Issue 31.

Eberhart, H. D. (1947) Fundamental studies of human locomotion and other information relating to design of artificial limbs, Prosthetic Device Research Project, Univ. of California, Berkeley, California, National Library of Medicine, WE172qN278F.

Flowers, W. C. (1972) A man-interactive simulator system for above-knee prosthetics studies, Ph.D. Thesis, Department of Mechanical Engineering, MIT, Cambridge, MA.

Flowers, W. C. and Mann, R. W. (1977) An electrohydraulic knee-torque controller for a prosthesis simulator. J. biomech. Engng 99, k(1), 3-8.

Frigo, D. and Rodano, R. (1982) Comparison between the reactive moments at the lower limb joints of normal and prosthetized subjects. Proceedings of an IF AC Symposium on Control Aspects of Prosthetics and Orthotics, Ohio State University.

Grimes, D. L. and Flowers, W. C. (1982) Multi-mode aboveknee prosthesis controller, IFAC Control Aspects of Prosthetics and Orthotics, pp. 43-53.

Grimes, D. L. (1979) An active multi-mode above-knee prosthesis controller, Ph.D. Thesis, Department of Mechanical Engineering, MIT, Cambridge, MA

Judge, G. B. (1980) Survey of knee mechanisms for artificial legs, biomechanical Research and Development Unit, Roehampton Lanc, London, Revised.

Stein, J. L. (1983) Design issues in the stance phase control of above-knee prostheses, Ph.D. Thesis, Department of Mechanical Engineering MIT, Cambridge, MA.

Stein, J. L. and Flowers, W. C. (1984) A system for measuring the three-dimensional gait of an above-knee amputee wearing a prosthesis simulator, Second International Conference on Rehabilitation Engineering, Ottawa, Canada Rehabilitation Engineering Society of North America.

Winter, D. A., Quanbury, A. O., Hobson, D. A., Sidwall, H. G., Reimer, G., Trenholm, B. G., Steinke, T. and Shlosser, H. (1974) Kinematics of normal locomotion-a statistical study based on television data. J. Biomechanics 7, 479-486. 